\title{
Forensic facial reconstruction under test
}

\begin{abstract}
Forensic facial reconstruction - rebuilding the face of an individual from skeletal remains for the purpose of identification is, of course, the most unscientific of all the vast array of forensic techniques now available to aid the identification of an individual, but sometimes, especially concerning skeletal remains buried for long periods, a facial reconstruction can still be helpful. I do not intend this paper to be a technical overview on the methodology of facial reconstruction, it centres on the interesting proposition: if several people carried out a facial reconstruction of the same person, would the end products look like each other and, would they be identifiable as the person in life whose face they were attempting to reconstruct? I came to facial reconstruction through my background in archaeology and so my reconstructions were of archaeological remains before I became interested in facial reconstruction techniques for forensic use. Having been asked by the University of Cambridge, UK if I would run a series of training courses at their Institute for Continuing Education at Madingley, I decided the courses would be a great way to test and refine reconstruction techniques. On each course 15 students were given an identical skull cast, tissue depth data set, tool kit, clay and step by step instructions. The big question was, if the course participants failed to produce reconstructions that were identifiable as the same person, what could be improved in terms of methodology to make the system of working more reliable?
\end{abstract}

Keywords: forensic facial reconstruction; skeletal remains; reconstruct face; skull cast; forensic artist; tissue depth
Volume I Issue I - 2015

\author{
Martin PWeaver \\ Freelance Forensic Anthropologist, Stonehaven Research, \\ Bulgaria \\ Correspondence: Martin PWeaver, Freelance Forensic \\ Anthropologist, Stonehaven Research, Kamenna Reka 6546, \\ Topolovgrad, Bulgaria, Tel +447860742968, \\ Email martinpweaver@gmail.com
}

Received: June 12, 2015 | Published: June 24, 2015

\section{Participants and hopes}

The students came from a wide variety of backgrounds, some relevant to the course subject and some not, they were of varying ages and abilities, some were artistic, some were not. I considered that these personal variations would highlight areas of the build system that were most subject to individual interpretation and therefore liable to be variable. Facial reconstruction does not meet the Daubert standard for scientific test repeatability, so is not used in a court of law as evidence in most countries and states. I hoped that if I could refine the method of working and produce a system that produced reliable and accurate results when followed by different forensic artists, forensic facial reconstruction could become more of a science than an art and could, possibly, one day be semi-automated using skull scans and computer software.

\section{Systems and methodology}

Over the four years I ran these courses we did try different systems, starting with the American system ${ }^{1}$ which involves fitting tissue depth pegs of measurement appropriate to the skull type (sex, age, ethnicity) and applying clay between the pegs at the same thickness and then filling in-between to produce a tissue depth layer over the skull that is then refined. This system seems quite basic and as it requires a high degree of artistic capability and anatomical knowledge to produce a credible end result, artistic subjectivity plays a large part and on my courses I found wide variations in the end product. I should point out here that that tissue depth pegs under normal circumstances are for guidance only as nutrition and basic health causes some individual facial variation. The tissue depth data sets are chosen for the subject and there are many available sets corresponding to the sex, age, ethnicity and weight of the subject, be they emaciated, normal or obese. For these courses I supplied a modified data set that matched the exact requirements of the subjects under reconstruction. Most forensic artists worldwide now use a version of the system developed in England by Richard Neave, in the 1970's at Manchester University and called the Manchester Method. ${ }^{2}$ In this system the muscles and soft tissues are modelled on the skull surface before applying a 'sheet' of $4 \mathrm{~mm}$ clay skin so the tips of the tissue depth pegs are just at the surface. This system looks very scientific especially in build progression photographs but the underlying facial musculature, glands and fatty deposits are unquantifiable in terms of their exact dimensions and, of course, do vary somewhat from face to face, even in faces from the same skull type, weight, age and sex. I found that the Manchester system of working caused the students to produce, for want of a better word 'lumpy' faces after the skinning process that still needed much refinement, somewhat negating the point of carefully modelling the sub-structure in the first place. However, neither system helps with soft tissue areas of the nose, eyes, mouth and ears and despite the course students' best efforts, occasionally some slightly grotesque and comedic results ensued.

\section{Soft tissue areas}

The proportions and detail of the soft tissue areas are gained by measurement and study of, in the case of the nose, the width/height/ shape of the nasal aperture and the angle of the nasal spine etc, The eye shape can be assessed by measurement and study of the shape of the eye sockets and the eyebrow shape determined by the shape of the supraorbital rim and brow ridge shape. The mouth shape is assessed by the measurement and study of the teeth. The ears are assessed by the angle of the jaw line. There is a wealth of research material to inform one as to the modelling of these areas which are, of course, dependant on race, age, sex and skull type. To help the students achieve uniformity of results and to minimise metrical analysis errors, the appropriate measurements and a step by step method of creating these soft tissue areas was provided. The procedures were improved, course on course, ending up with an improved system of reconstruction for 
the soft tissue areas with some elements of the Manchester and the American methods.

\section{Courses and outcomes}

We started the courses with archaeological subjects and by the end we were using a forensic sample skull copy with a photograph of the person in life; this was not shown to the students until the end of the course. I would say that a third of the fifteen students on the last course did manage to produce a reconstruction that had a good likeness to the subject and given more training in facial anatomy most of the students could also have achieved this. This run of courses was possibly the first time that facial reconstruction had been put to the test like this and it was most interesting to see where each different system's weak points were. Also of interest and quite unexpected by me, was the student demographic and how they reacted to the face developing before them and how some tried to unconsciously model the subject to their own way of wanting this person to look. For instance young female students would invariably endow the forensic subject (a 25year old woman) with a pouting mouth, completely hiding the tissue depth pegs for that area in extra flesh. This is just one indicator of artistic subjectivity which I found takes place when the reconstruction has been 'fleshed out' and the last stages of refining the soft tissue is taking place. I think in this situation; creating a face for the first time without knowing what the end product will look like, creates a natural desire to produce something aesthetically pleasing to the artist. We must bear in mind that the students that took part in these courses had never done anything like this before so were bound to make errors that hopefully a seasoned professional fully trained forensic artist would not. Interestingly, I did note that students from an art background generally produced the least accurate reconstructions, happy to disregard the supplied data somewhat to produce a face they were happy creating and were consequentially surprised when the photograph of the subject in life was revealed at the end of the course. People of middle age usually produced the best results, rigidly adhering to the data sets provided.

\section{Conclusion}

Students did achieve uniformity in the general shape of the face, it was the eyes, ears, nose and mouth that proved impossible for everyone to render accurately and this is the Achilles' heel of facial reconstruction; be it carried out by professionals or students. Whether it is carried out with modelling clay or by sophisticated computer programs such as Sensable and the like, these critical areas of the human face are difficult to get completely right in all cases as they are subject to artistic interpretation due to the impossibility of creating exact data sets. The human face does not always conform to even average data sets and small variations here and there can make all the difference to a successful identification as humans are superbly adapted to facial recognition and even small deviations can deter recognition. So I must report that sadly by the end of the last course run in 2011, having developed a reliable system of working, we were unable to achieve anything like uniformity in all reconstructions and were a long way off achieving the Daubert standard which in my view will never be achieved. As forensic facial reconstruction is essentially part science, part art, it can never be deemed to be a scientific process but this isn't necessarily a bad thing. Forensic artists with the necessary knowledge of Anthropology and facial anatomy can and do produce credible results, often able to aid the identification of a missing person.

\section{Acknowledgments}

None

\section{Conflicts of interest}

The author declares that there are no conflicts of interest.

\section{References}

1. Snow CC, Gatliff BP, McWilliams KR. Reconstruction of facial features from the skull: an evaluation of its usefulness in forensic anthropology. Am J Phys Anthropol. 1970;33(2):221-228.

2. Wilkinson CM. Forensic Facial Reconstruction. International Journal of Osteoarchaeology. 2004;292. 